\title{
Four Graph Partitioning Algorithms
}

\author{
Fan Chung Graham \\ University of California, San Diego \\ fan@math.ucsd.edu
}

\begin{abstract}
We will discuss four partitioning algorithms using eigenvectors, random walks, PageRank and their variations. In particular, we will examine local partitioning algorithms, which find a cut near a specified starting vertex, with a running time that depends on the size of the small side of the cut, rather than on the size of the input graph (which can be prohibitively large). Three of the four partitioning algorithms are local algorithms and are particularly appropriate for applications arising in connection with Webgraphs and Internet economics.
\end{abstract}

